\title{
Control of an Isolated Table's Fluctuation Caused by Supplied Air Pressure Using a Voice Coil Motor*
}

\author{
Habiburahman SHIRANI** and Shinji WAKUI** \\ ${ }^{* *}$ Tokyo University of Agriculture and Technology \\ 2-14-16 Naka-cho, Koganei, Tokyo,184-8588, Japan \\ E-mail:wakui@cc.tuat.ac.jp
}

\begin{abstract}
Pneumatic type anti-vibration apparatuses are used in the field of semiconductor manufacturing and precision measurement. The variation of the supplied air pressure from the air compressor causes the position fluctuation of the isolated table. A control method using a voice coil motor (VCM) as the actuator is proposed in this study to control the position fluctuation of the isolated table caused by the supplied air pressure. The feedforward compensator control scheme is used to provide a proper controlled signal to the VCM. According to the controlled signal, VCM exerts driving force in the opposite direction of the air spring expansion or compression to suppress the vibration of the isolated table.
\end{abstract}

Key words: Voice Coil Motor, Pneumatic Anti-Vibration Apparatus, Supplied Air Pressure, Isolated Table, Feedforward Compensator

\section{Introduction}

Active pneumatic type anti-vibration apparatuses are widely used in the field of semiconductor manufacturing, precise measurement and positioning. The isolated table of the pneumatic anti-vibration apparatus is supported by the air spring. The air spring is actively controlled by servo valve to attenuate the vibration transmitted from the floor and to suppress the vibration on the table. The air compressor supplies compressed air to the air spring's chamber. The pressure of the compressed air varies during the compression process. The pressure of the compressed air linearly increases from low pressure to high pressure and also linearly decreases from high to low pressure. In other words, the pressure of the supplied air varies with respect to time. This variation of the pressure causes the position fluctuation of the isolated table. Since the air compressor periodically compresses the air, the variation of the supplied air pressure causes periodic fluctuation of the isolated table. In order to control the variation of the supplied air pressure, multi-connections' air regulators are often used. However, the multi-connections' air regulator leads to the pressure loss of the compressed air. In addition, the multi-connections' air regulator cannot completely suppress the variation of the supplied air pressure.

In references (1) and (2), we used air springs as the actuator to control the position fluctuation of the isolated table caused by the supplied air pressure. The driving force of the air spring is larger than VCM; however, the driving force resolution, accuracy and acceleration of the VCM are higher than air spring. Active pneumatic anti-vibration apparatuses for semiconductor manufacturing and measurement apparatuses have required even higher performance because the line width of the semiconductor continues to decrease. Therefore, the current study proposes a method of using VCM as the actuator with feedforward compensator to precisely control and suppress the position fluctuation of the

[DOl: 10.1299/jsdd. 4009 (No. 09-0743)

Copyright $(C 2010$ by JSME 
isolated table caused by the supplied air pressure.

The remainder of this paper is organized as follows. The experiment setup, and the parameters and variables used in the control block diagram, including the block diagram of anti-vibration apparatus and the calculation of the feedforward compensator, are briefly described in Section 2. The discussion of the experiment results is presented in Section 3. The comparison of VCM and air spring as actuators is discussed in Section 4. The switching of the feedforward compensator and the control scheme to reduce the switching effect on the isolated table are given in Section 5. Concluding remarks are provided in Section 6.

\section{Pneumatic anti-vibration apparatus}

\subsection{Experimental setup}

Fig. 1 shows the experimental setup of the pneumatic anti-vibration apparatus. The isolated table is mounted on the air spring, weighs about $120 \mathrm{~kg}$, and has an acceleration sensor on its top. A pressure sensor which measures the inner pressure of the air spring is connected to the air spring. A position sensor which detects vertical displacement of the isolated table is installed on one side to the bottom of the hung frame of the isolated table. VCM is installed beneath the isolated table's hung frame. The moving magnetic part of the VCM is connected to the bottom of the isolated table's hung frame and the static coil part is fixed to the base of the anti-vibration apparatus. VCM gets power from the VCM driver. Its driver receives a controlled signal from the DSP controller for its excitation to provide proper current. The VCM moving part moves and exerts a driving force on the isolated table in the opposite direction to the air spring's expansion and compression.

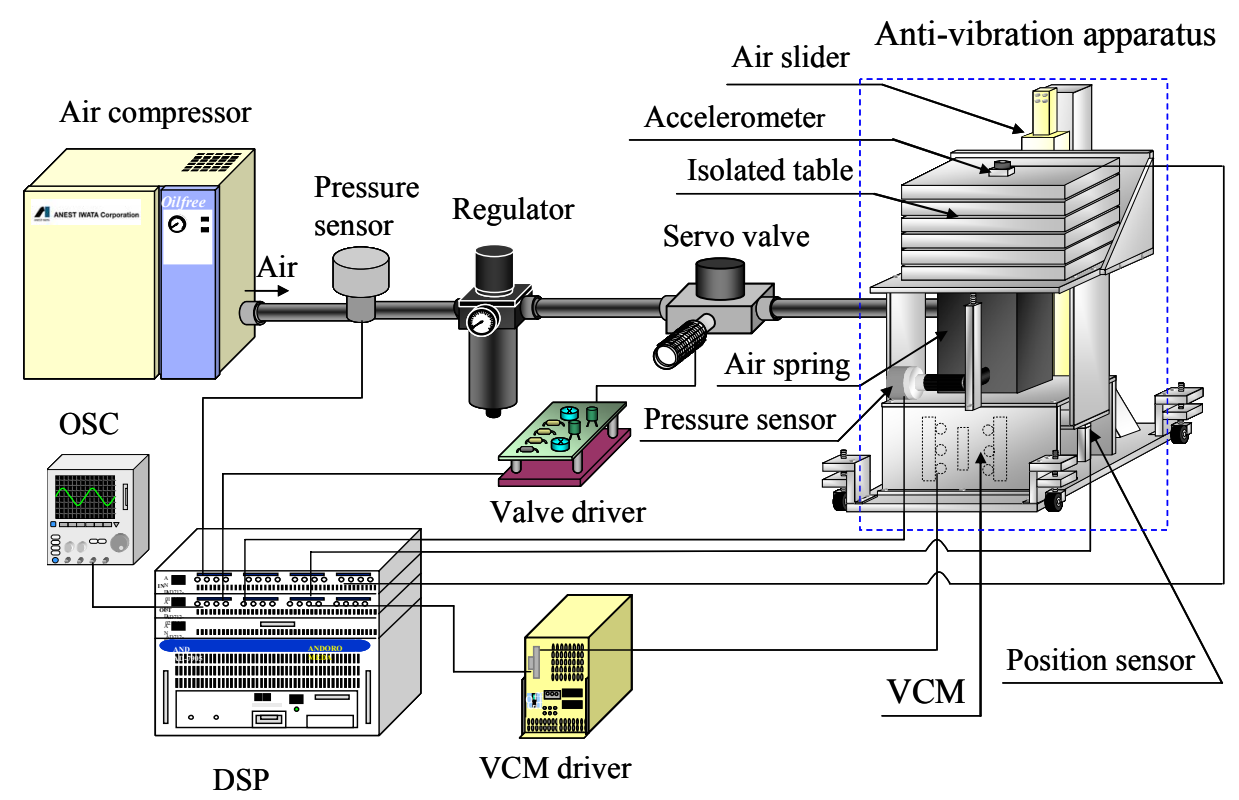

Fig.1 Experimental setup of the pneumatic anti-vibration apparatus

\subsection{Control system}

The objective of this study is to control the position fluctuation of the isolated table caused by the supplied air pressure. The block diagram of the pneumatic anti-vibration apparatus, including the controller, is shown in Fig. 2. The thick tabulated block represents the proposed feedforward compensator that will be discussed in the coming section. The desired vertical displacement of the isolated table $x_{r e f}$ is given as a reference input signal. The proportional gain $k_{s}$ is used to maintain the displacement of the isolated table, and the acceleration PI is implemented to control the damping ratio of the isolated table. The pressure PI is employed to keep the inner pressure of the air spring constant. The parameters and variables used in the block diagram are defined as follow, $M$ : mass $[\mathrm{kg}], d$ : viscous 
damping coefficient $[\mathrm{N} \cdot \mathrm{s} / \mathrm{m}], k$ : stiffness constant $[\mathrm{N} / \mathrm{m}], A_{0}$ : received area $\left[\mathrm{m}^{2}\right], \beta_{0}$ : compressibility $[1 / \mathrm{Pa}], V_{0}$ : air spring volume $\left[\mathrm{m}^{3}\right], G_{q}$ : flow gain of the servo valve $\left[\mathrm{m}^{3} / \mathrm{s} / \mathrm{V}\right]$, $c$ : flow conductance $\left[\mathrm{m}^{3} / \mathrm{s} / \mathrm{Pa}\right], k_{\text {pos }}$ : position sensor's detection sensitivity $[\mathrm{V} / \mathrm{m}], k_{g}$ : feedback pressure's gain [V/Pa], $k_{p r}$ : pressure PI gain [V/V], $T_{p}$ : time constant of pressure PI compensator [s], $k_{a}$ : acceleration's feedback gain $\left[\mathrm{V} / \mathrm{m} / \mathrm{s}^{2}\right], k_{a r}$ : forward gain of acceleration feedback $[\mathrm{V} / \mathrm{V}], T_{a}$ : time constant of acceleration PI compensator [s], $k_{s}$ : position gain $[\mathrm{V} / \mathrm{V}], x$ : displacement of isolated table $[\mathrm{m}], x_{0}$ : displacement of floor vibration $[\mathrm{m}], w$ : input voltage $[\mathrm{V}], p$ : pressure $[\mathrm{Pa}], d_{p r}$ : pressure disturbance $[\mathrm{Pa}], d_{f}$ : flow disturbance $\left[\mathrm{m}^{3} / \mathrm{s}\right]$, $k_{V C M}$ : VCM driver gain [N/V], $G_{f}(s)$ : feedforward compensator [V/Pa], $G_{d}(s)$ : disturbance transfer function from $d_{p r}$ to $d_{f}\left[\mathrm{~m}^{3} / \mathrm{s} / \mathrm{Pa}\right]$.

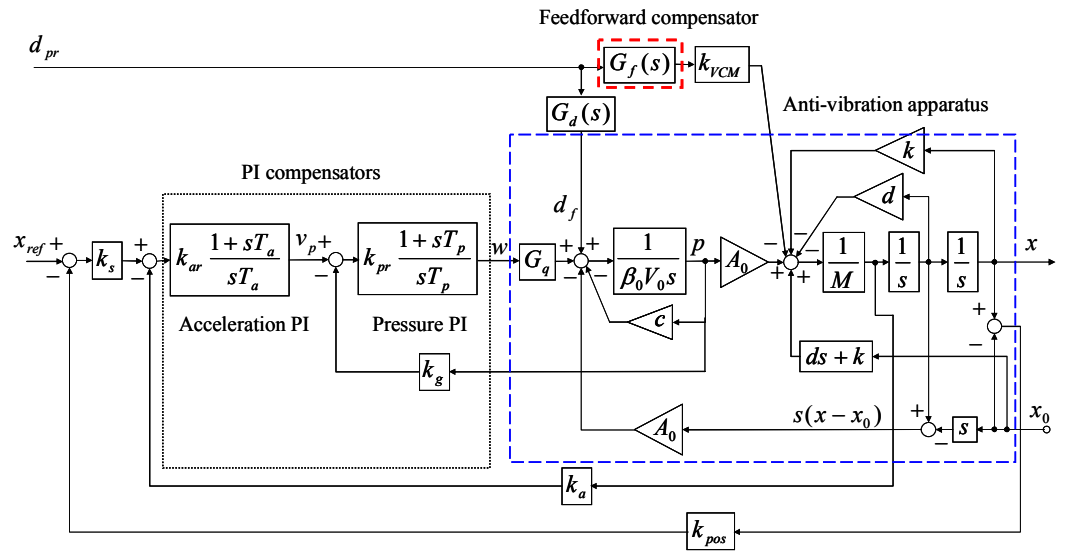

Fig. 2 Control block diagram of active pneumatic anti-vibration apparatus

\subsection{Design of $G_{f}(\mathrm{~s})$}

Referring to Fig. 2, the feedforward compensator is employed to provide a control signal to the VCM. $G_{f}(s)$ is the transfer function of the feedforward compensator. Prior to the design of $G_{f}(s)$, it is essential to find the relation of the variables used in the block diagram of the anti-vibration apparatus including the proposed feedforward compensator. Eq. (1) below represents the relationship of $x, x_{0}, w$, and $d_{p r}$.

$$
\begin{aligned}
& \left(G_{q} w+G_{d}(s) d_{p r}-A_{0}\left(x-x_{0}\right) s\right) \frac{A_{0}}{\beta_{0} V_{0} s+c}-G_{f}(s) k_{V C M} d_{p r}+(d s+k) x_{0}-d x s-k x \\
& =M x s^{2}
\end{aligned}
$$

The variation of the supplied air pressure is actually disturbance to the isolated table. The transfer function $G_{f}(s)$ should be designed in such a way which can provide a proper controlled signal to the VCM to suppress the disturbance. To reduce the disturbance to zero, in this case, the following equality should satisfy:

$$
G_{f}(s)=\frac{A_{0}}{k_{V C M}} \cdot \frac{1}{\beta_{0} V_{0} s+c} G_{d}(s)
$$

In the case of choke flow, let $G_{d}(s)$ be $K$ which can be any constant. Thus we have,

$$
G_{f}(s)=\frac{A_{0} K}{k_{V C M}} \cdot \frac{1}{c\left(\frac{\beta_{0} V_{0}}{c} s+1\right)}
$$

If we set $A_{0} K / c k_{V C M}$ as $k_{f f(V C M)}$ and $\beta_{0} V_{0} / c$ as $T$, then the following equation can be obtained,

$$
G_{f}(s)=k_{f f(V C M)} \frac{1}{T s+1}
$$

The pressure of the supplied air shown in Fig. 3, increases with almost constant slope for sometime and then decreases with constant slope for the remaining time of one period. The slope of increasing part of the supplied air pressure signal is greater than decreasing part 
and it is the main concern which causes the fluctuation of the isolated table. Therefore, it can be treated as a ramp signal and the $s$-domain form of the ramp signal is expressed as $d_{p r 0} / s^{2}$. Applying this ramp signal to the feedforward compensator, the output is:

$$
v_{\text {out }}=k_{f f(V C M)} \frac{1}{T s+1} \cdot \frac{d_{p r 0}}{s^{2}}
$$

The time constant $T=\beta_{0} V_{0} / c=8.935[\mathrm{~s}]$ is a longer time constant, therefore, $1 /(T S+1)$ becomes almost an integrator. When the supplied air pressure signal which is a ramp signal, applies to the integrator, the integrator magnifies and converts it to a curved signal which can be provided to the VCM. VCM is very sensitive to the exciting signal, a small change in the exciting signal results a significant change in the driving force of the VCM. Also, based on the improper compensator's signal, the VCM driver might allow a heavy current to flow through the VCM coil which causes a risky accident to the VCM. Therefore, it is needed to transform Eq. (4) to the form in order to realize fine tuning and safety of the VCM. In this case, Eq. (4) should be transformed to a form to provide static and dynamic compensations. Thus, $G_{f}(s)$ should be improved and expressed as,

$$
G_{f}(s)=k_{f f(V C M)}\left(1-\frac{T s}{T s+1}\right)
$$

The right side of Eq. (6) comprises a static and a dynamic terms. In order to provide a proper controlled signal to the VCM, the static term and the coefficient of the dynamic term, $k_{f f(V C M)}$ of Eq. (6) should be tuned separately, starting from smaller to larger parameters. Therefore, the static term is named as $k_{1}$ and the coefficient of the dynamic term is named $k_{2}$, thus $G_{f}(s)$ can be expressed as follows,

$$
G_{f}(s)=\left(k_{1}-k_{2} \frac{T s}{T s+1}\right) \cdot k_{3}
$$

where $k_{1}$ and $k_{2}$ are separately tunable gains, and $k_{3}$ is a constant switching gain (1or 0 ). Fig. 4 shows the block diagram of the feedforward compensator based on Eq. (7).

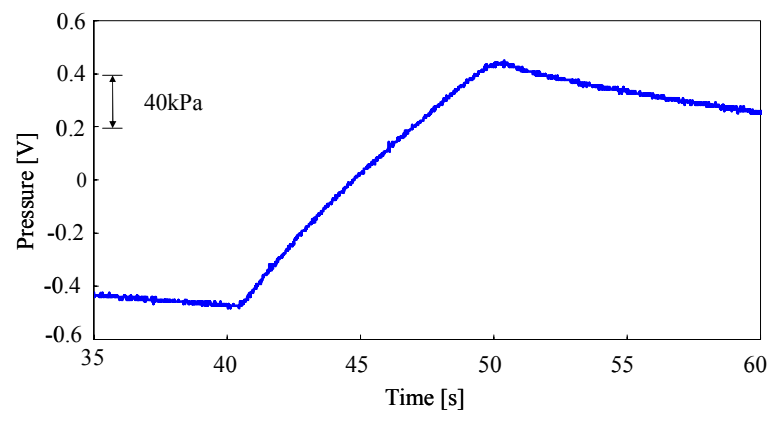

Fig. 3 Supplied air pressure

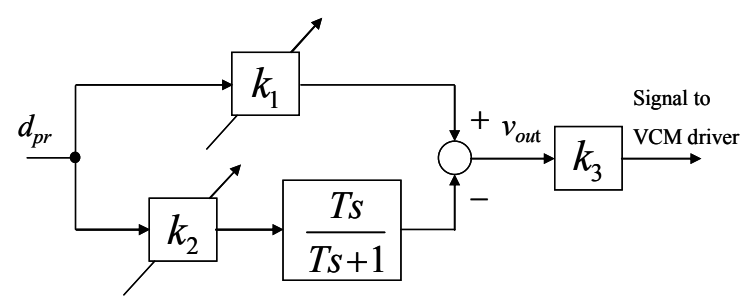

Fig. 4 Block diagram of the feedforward compensator based on Eq. (7).

\section{Experiment results}

\subsection{Results of tuning the $k_{1}$ and $k_{2}$ parameters}

The tuning method of the $k_{1}$ and $k_{2}$ parameters of the feedforward compensator was based on trial and error procedure. $k_{2}$ was set to a specific smaller parameter and $k_{1}$ was 
increased from smaller to larger parameters and the fluctuation of the isolated table was continuously monitored. Then $k_{1}$ was kept fixed and $k_{2}$ was increased from smaller to larger parameters and the fluctuation of the isolated table was observed. It was realized that by setting both $k_{1}$ and $k_{2}$ to the same parameters, the fluctuation of the isolated table continued to decrease. Fig. 5 shows the experiment result of the displacement of the isolated table while setting both $k_{1}$ and $k_{2}$ to 0.3 and then to 0.62 . Finally, we increased both $k_{1}$ and $k_{2}$ starting from 0.62 to larger parameters. Eventually, it was realized that by setting both $k_{1}$ and $k_{2}$ to 0.73 , the fluctuation of the isolated table was almost completely suppressed and controlled. We farther increased both $k_{1}$ and $k_{2}$ beyond 0.73 ; it was observed that the result of the control of the isolated table's fluctuation was again degraded. Therefore we concluded that 0.73 is the optimal parameter for both $k_{1}$ and $k_{2}$. Fig. 6 shows the result of the optimal tuning parameter.

The result of the experiment showed that by tuning both $k_{1}$ and $k_{2}$ to 0.73 , the position fluctuation of the isolated table was suppressed. The relation $k_{1}=k_{2}$ is also theoretically expected from Eq. (6). Thus the experiment result coincided with theoretical result.

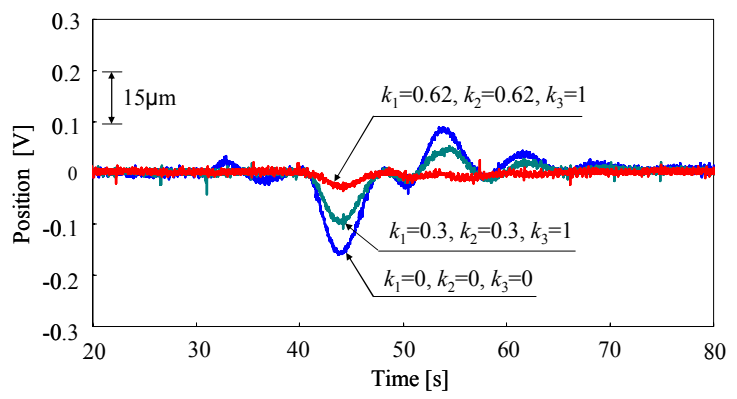

Fig. 5 VCM effect on the isolated table's fluctuation

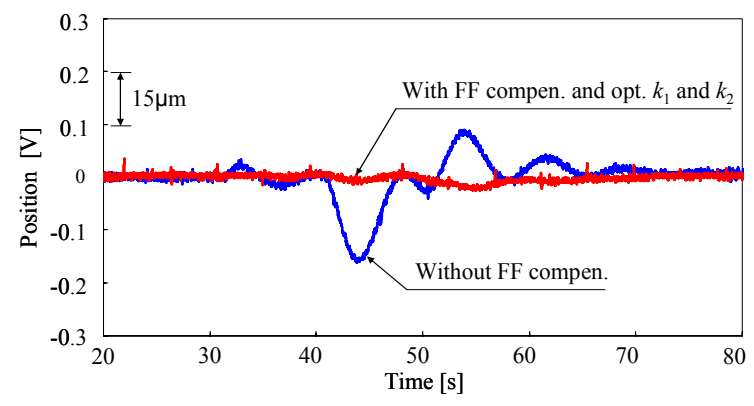

Fig. 6 Result of the precise tuning of $k_{1}$ and $k_{2}$

\subsection{Pressure impact drop}

Pneumatic anti-vibration apparatuses are employed for semiconductor manufacturing. For example, the lithography machine can be set on the isolated table during semiconductor's wafer exposure process. Sometimes due to some kind of an accident which is a natural occurrence at the factory, something may cause to drop the pressure of the anti-vibration apparatus. Dropping the pressure of the pneumatic anti-vibration apparatus is known as the pressure impact drop. Such kind of pressure impact drop would result in the fluctuation of the lithography machine set on the isolated table which would lead to damage the whole exposure process.

To make a simulation of the industrial pressure impact drop, an experiment is performed while manually closing the air flow from the air compressor to the air spring for a moment and checking the pressure impact drop effect on the position of the isolated table. The left side of Fig. 7 shows the effect of the pressure impact drop on the isolated table's 
position when the control signal of the feedforward compensator is not applied to the VCM. It is clear from the left side of Fig. 7 that the pressure impact drop fluctuated the isolated table. However, the right side of Fig. 7 shows the result of the position of the isolated table when feedforward compensator provided the controlled signal to the VCM, and $k_{1}$ and $k_{2}$ were set to their optimal values i.e. 0.73 . It is obvious from the experimental result that when feedforward compensator provided the controlled signal to the VCM, the position of the isolated table was kept stable against pressure impact drop. Thus we can conclude that the feedforward compensator with the VCM as an actuator can maintain the stability of the isolated table caused by the pressure impact drop.

It is worth mentioning to notice here that in practice, the performance of the feedforward controller can be affected by the parameters' variation of the process. However, the performance degradation of the feedforward controller in the current experiment was not observed. Because, the feedback controllers shown in Fig. 2 were set and optimized to completely control the parameters' variation of the anti-vibration apparatus.

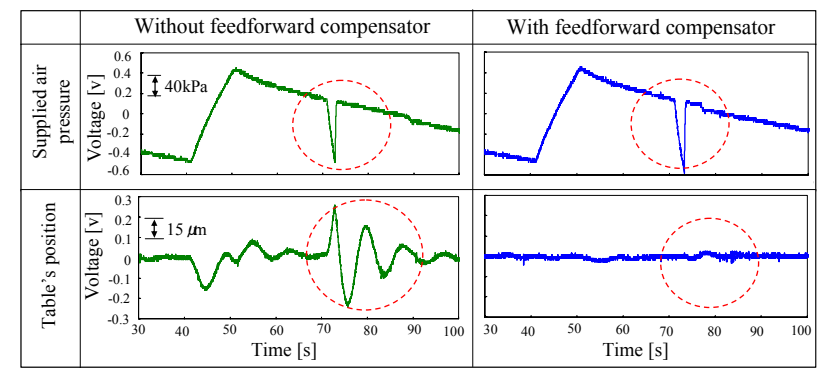

Fig. 7 Experiment result of the pressure impact drop

\section{Comparison of the air spring with VCM}

\subsection{Air spring with feedforward controller}

Several kinds of actuators can be used for active anti-vibration apparatus such as mechanical mechanism, piezoelectric actuators, air springs, electromagnetic motors and linear motors. The isolated table is supported by the air spring which consists of an air filled chamber, an elastomeric diaphragm and a piston. The air spring is actively controlled by a servo valve to attenuate the vibration of the isolated table ${ }^{(3)}$.

The air spring was used as the actuator with the feedforward compensator by the authors in references (1) and (2) to control the isolated table's fluctuation caused by the supplied air pressure. The advantages of the air spring include that it can generate a larger force and stroke. It does not generate heat and is intrinsically safe. The disadvantages of the air spring are: low accuracy and longer response time. Air spring's force resolution is low; the approximate force resolution of the air spring used in the experiment of the current study is about $300 \mathrm{gf}$ while assuming a $10 \mathrm{mV}$ noise level. The linearity is also not good. Therefore, air spring can be used in the applications where large drive force and stroke are demanded. The authors used the control block diagram with feedforward compensators as shown in Fig. 8.

Several feedforward compensators $G_{f f 14}(s)$ that are shown in the block diagram of Fig. 8 were developed. The effective feedforward compensator is expressed in Eq. (8) below which provided a better controlled signal to control the position fluctuation of the isolated table.

$$
G_{f f 4}(s)=\frac{1}{G_{q}} \cdot G_{d}(s) \rightarrow k_{f f} \text { (Constant value) }
$$

The feedforward compensator $G_{f f 4}(s)$ is a proportional feedforward controller. Using the 
feedforward compensator of Eq. (8), the experiment result of the position of the isolated table is shown in Fig. 9.

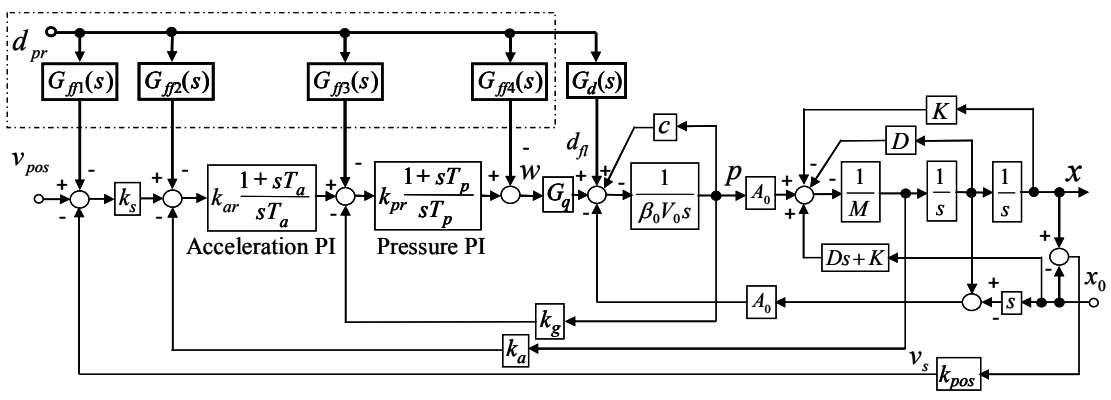

Fig. 8 Schematic control diagram of active anti- vibration apparatus ${ }^{(1)}$

Fig. 10 shows the experiment result of the pressure impact drop. It is obvious that using air spring, the position fluctuation of the isolated table caused by pressure impact drop is reduced; however the fluctuation of the isolated table is not completely suppressed.

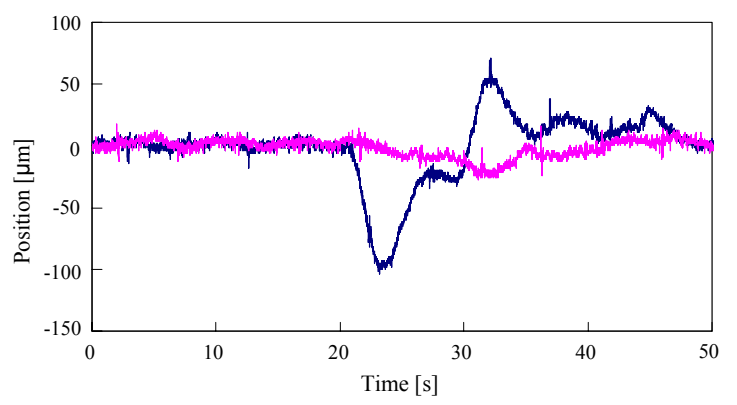

Fig. 9 Result of position signal based on Eq. (8) ${ }^{(1)}$

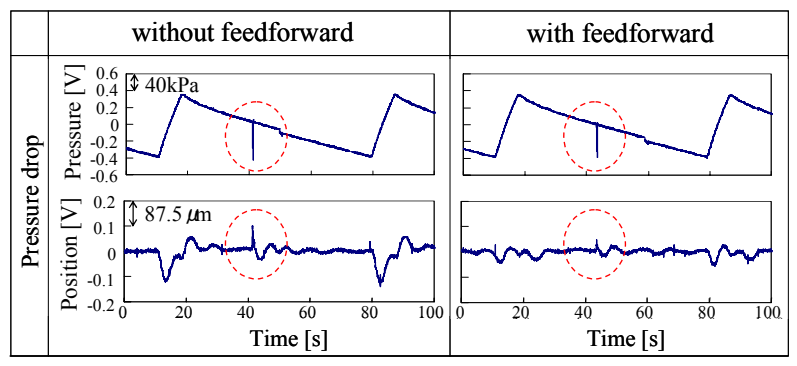

Fig. 10 Experiment result of the pressure impact drop ${ }^{(1)}$

\subsection{VCM with feedforward controller}

VCM is a linear motor which is used as a linear actuator. The main advantages of the VCM are: The ability to generate force with very high accuracy over a limited range of travel ${ }^{(4)}$; good linearity between the coil current and the thrust ${ }^{(5)}$; fast response time; and high force resolution. The approximate force resolution of the VCM including the driver used in the experiment setup of the current study is $30 \mathrm{gf}$, assuming $10 \mathrm{mV}$ noise level. However, the disadvantages of the VCM are: the small force and stroke; and VCM coil generates heat and is intrinsically not safe. Based on the above mentioned advantages, VCM can be used in the applications where highly precise positioning is required.

The feedforward compensator to the VCM expressed in Eq. (7), comprises two main parameters, gains $k_{1}$ and $k_{2}$, and the time constant $T$. According to theoretical calculation, the feedforward compensator can be obtained as expressed in Eq. (4); however, for the safety of the VCM, Eq. (7) was derived for fine tuning the gain parameters. During the experiment, many 
different tuning parameters were set to $k_{1}$ and $k_{2}$. However, as according to the theory when the same parameter i.e. 0.73 was set to both $k_{1}$ and $k_{2}$, the position fluctuation was suppressed. Therefore, 0.73 was known as the optimal tuning parameter for $k_{1}$ and $k_{2}$.

Comparing the experiment results shown in Figs. 6 and 9, it can be claimed that using VCM, position fluctuation of the isolated table is more precisely controlled. Also, by comparing the experiment result shown in Figs. 7 with the result shown in 10, we can conclude that using VCM with feedforward compensator, the position fluctuation of the isolated table caused by pressure impact drop is more accurately suppressed. It is worth mentioning that the experiment setup used for the experiment of the air spring is mutually independent from the experiment setup used for the VCM experiment. However, the same sensors, air springs, and VCM were used for both sets of anti-vibration apparatuses.

\section{Feedforward compensator's switching}

Pneumatic anti-vibration apparatus is used in the industry for manufacturing the semiconductor devices. It is typical in the industry to start or stop the pneumatic anti-vibration apparatus. Sometimes an accident also takes place which leads the apparatus to be stopped. Besides the feedforward controller, there would be other kinks of feedback controllers to control the floor and stage vibrations transmitted to the isolated table. Therefore, the sequence of the application of the controllers should be considered while starting or stopping or when an accident occurs. The isolated table of the pneumatic antivibration apparatus compared to electric systems needs longer settling time to come to the steady-state. Therefore, during starting or stopping or during an accident, if both feedforward and feedback controllers are simultaneously applied to provide the controlled signals it would lead to longer the settling time. To avoid the longer settling time which sometime results in damage to anti-vibration apparatus, it is suggested that when starting the apparatus, first, the feedback loop controller should be activated and when the isolated table comes to the steady state, then the feedforward controller should be activated. While stopping the apparatus, firstly, the feedforward controller should be turned off. The same sequence should be followed for an accident too, i.e., first the feedforward controller and then the other feedback controller should be turned off.

Therefore, the feedforward compensator should be connected by an on/off switch to the VCM and switch on or switch off the feedforward compensator to the VCM. Fig.11 illustrates the concept of switching of the feedforward compensator. When the switch command is set to 0 , the feedforward compensator is disconnected from the VCM driver and by setting the switch command to 1 , the feedforward compensator is connected to the VCM.

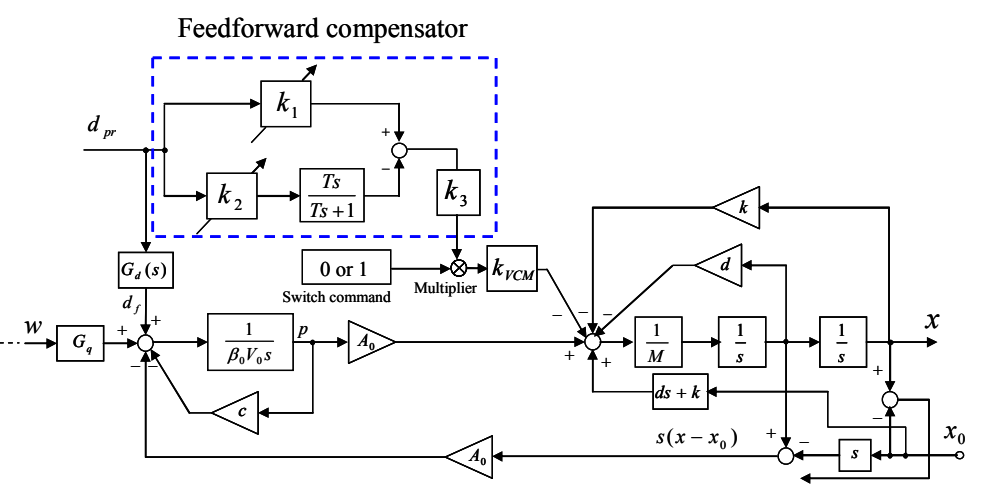

Fig. 11 Control block diagram with feedforward compensator's switching

On the other hand, the feedforward compensator's switching also causes destabilization and fluctuation of the position of the isolated table. Fig. 12 shows the feedforward 
compensator's switching effects on the position of the isolated table. The experiment was performed for the two cycles of the supplied air pressure. It is clear from the result that the feedforward compensator's switching causes the position fluctuation of the isolated table.

In order to reduce the fluctuation of the isolated caused by switching of the feedforward compensator, a feedback damping loop is considered to be a control scheme. The accelerometer at the top of the isolated table measures the acceleration of the table's fluctuation and provides the signal through an integrator and a gain to the summer. The integrator and gain at the feedback loop are used to provide a damping signal to the VCM to generate damping force. Some semiconductor manufacturers select air spring to produce damping force and some others choose VCM. In the experiment of the current study, we selected VCM for the generation of the damping force. The summer adds the two signals from the feedforward compensator and the feedback damping signal. The output of the summer is fed to the VCM driver. During the fluctuation period of the table, the damping force of the VCM results to reduce the position fluctuation of the table. Fig. 13 shows the complete diagram including the feedback damping control scheme and the anti-vibration apparatus.

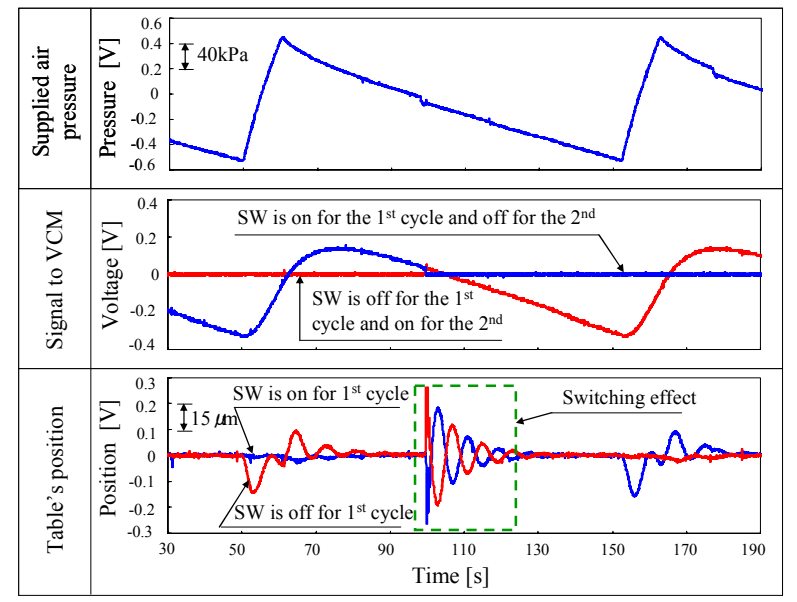

Fig. 12 Feedforward compensator's switching effect on the isolated table's position

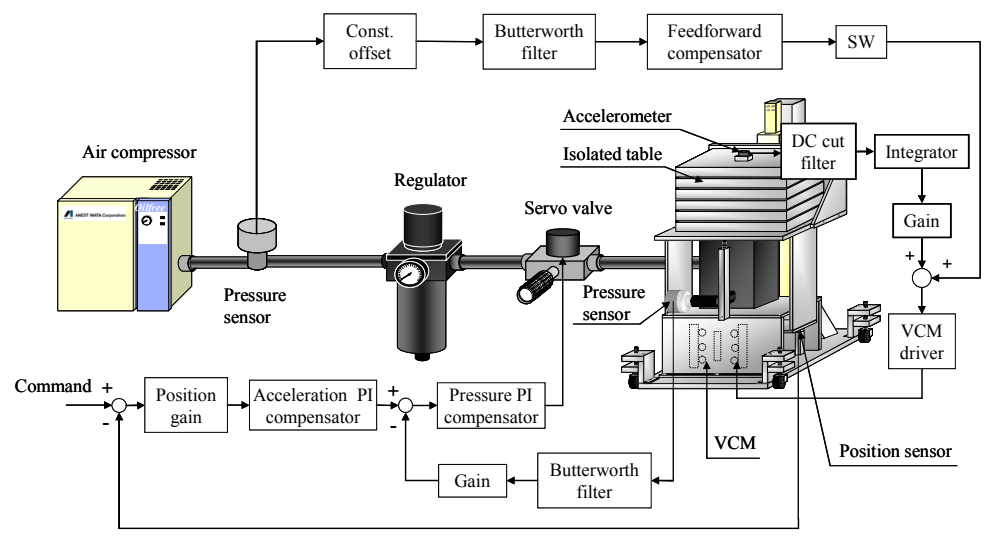

Fig. 13 Feedback damping loop control scheme to control the switching of the feedforward compensator

Applying the control scheme shown in Fig. 13, the experiment was performed and the result is shown in Fig. 14. By comparing the result of Fig. 12 with the result shown on Fig. 14, it is clear that the position fluctuation of the isolated table caused by the switching of the feedforward compensator is considerably reduced. Thus using the feedback damping loop 
resulted to reduce and attenuate the position fluctuation of the isolated table caused by feedforward compensator's switching.

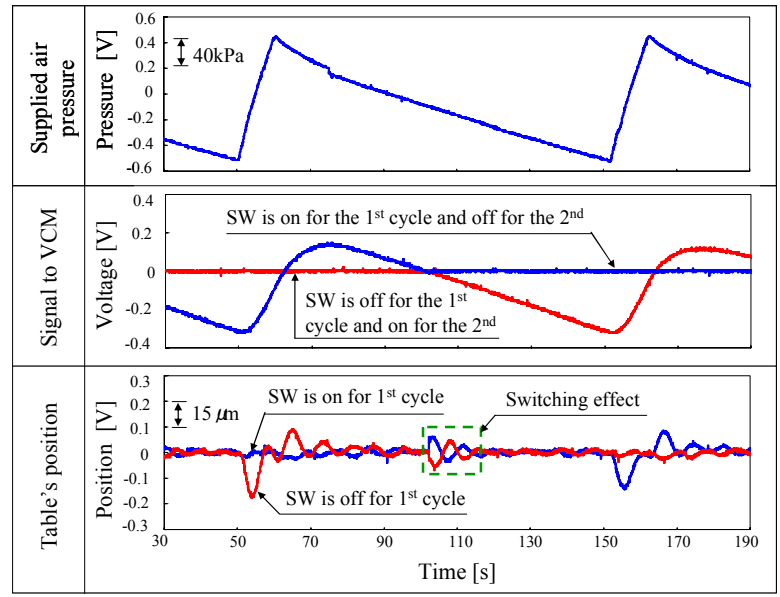

Fig. 14 Experiment result of the control of the fluctuation of the isolated table caused by switching of the feedforward compensator

\section{Summery}

The results of this study are summarized as follows:

(1) The variation of the supplied air pressure from the air compressor causes the fluctuation of the isolated table. VCM would work as a more efficient actuator with feedforward compensator to reduce and suppress the position fluctuation of the isolated table caused by supplied air pressure.

(2) The feedforward compensator with VCM as an actuator resulted to maintain the stability of the isolated table caused by the pressure impact drop

(3) The feedforward compensator with VCM as the actuator resulted to reduce the position fluctuation of the isolated table caused by the feedforward compensator switching.

\section{References}

(1) S. Wakui, K. Uryu, M. Takahashi and K. Yamamoto: Feedforward Control of Supplied Air Pressure and Air Flow Fluctuations for Pneumatic Type Anti-Vibration Apparatus, The Japan Society for Precision Engineering Vol. 73, No. 11, (2007), pp. 1215 - 1219.

(2) S. Wakui, K. Uryu, M. Takahashi and K. Yamamoto: A Suppression Method of Hunting due to Switching of Feedforward Compensation Based on Supplied Air Pressure Fluctuation, The Japan Society for Precision Engineering Vol. 74, No. 9, (2008), pp. $981-985$.

(3) K. Kawashima, T. Kato, K. Sawamoto and T. Kawaga: Realization of virtual sub chamber on active controlled pneumatic isolation table with pressure differentiator, Precision Engineering 31 (2007), pp. 139 - 145.

(4) R. Banik and D.-G. Gweon: Design and optimization of voice coil motor for application in active vibration isolation, Sensor and Actuator A 137 (2007), pp. 236 $-243$.

(5) Y.-D. Chen, C.-C. Fuh, and P.-C. Tung: Application of Voice Coil Motors in Active Dynamic Vibration Absorbers, IEEE Transactions on Magnetics, Vol. 41, No. 3, March (2005), pp. 1149 - 1154.

(6) T. Miyajima, T. Fujita, K. Sakaki, K. Kawashima and T. Kagawa: Development of a digital control system for high-performance pneumatic servo valve, Precision Engineering 31 (2007), pp. $156-161$. 